TRANSACTIONS OF THE

AMERICAN MATHEMATICAL SOCIETY

Volume 349, Number 8, August 1997, Pages 3343-3352

S 0002-9947(97)01901-6

\title{
EXTENSIONS OF MODULES OVER WEYL ALGEBRAS
}

\author{
S. C. COUTINHO
}

\begin{abstract}
In this paper we calculate some Ext groups of singular modules over the complex Weyl algebra $A_{n}$. In particular we determine conditions under which Ext is an infinite dimensional vector space when $n=2$ or 3 .
\end{abstract}

\section{INTRODUCTION}

A very important result of $\mathcal{D}$-module theory states that Ext groups of holonomic modules are finite dimensional. In this paper we investigate the behaviour of Ext groups for modules over the Weyl algebra which are generalizations of holonomic modules, the singular modules, defined by I.N. Bernstein and V. Lunts in [1]. These modules have representation theoretic properties very similar, in many ways, to the holonomic modules.

The most important geometric invariant of a $\mathcal{D}$-module is its characteristic variety. A holonomic module is a finitely generated $\mathcal{D}$-module whose characteristic variety is Lagrangian. Similarly, a singular module is defined in terms of its characteristic variety. In [1] and [12], it is shown that there exist singular modules whose characteristic varieties are hypersurfaces. This is the case discussed in $\S 2$.

In $\S 3$, we show that there exist singular modules of codimension 2 and study some of their properties. These results can be summed up by saying that if $M$ and $N$ are singular $A_{3}$-modules and if $\operatorname{codim}(M)<3$, then $\operatorname{Ext}^{1}(M, N)$ can be an infinite dimensional vector space. A few open problems are discussed in the final section.

I wish to thank J.T. Stafford for his many useful suggestions. During the preparation of this paper I was partially supported by a grant from $\mathrm{CNPq}(\mathrm{Brazil})$.

\section{Singular MODULES}

Let $A_{n}$ be the $n$th complex Weyl algebra. It is generated by the coordinate functions $x_{1}, \ldots, x_{n}$ and by the corresponding partial differential operators, which will be denoted by $\partial_{1}, \ldots, \partial_{n}$. The Bernstein filtration of $A_{n}$ is obtained by giving degree 1 to $x_{1}, \ldots, x_{n}, \partial_{1}, \ldots, \partial_{n}$.

Let $S_{n}$ be the graded ring of $A_{n}$ associated to the Bernstein filtration. For $1 \leq i \leq n$ denote by $y_{i}$ the symbol of $x_{i}$, and by $y_{i+n}$ the symbol of $\partial_{i}$. Then $S_{n}$ is a polynomial ring in the $2 n$ indeterminates $y_{1}, \ldots, y_{2 n}$. The symbol map of degree $k$ is a linear map of vector spaces $\sigma_{k}: B_{k} \rightarrow S_{n}(k)$, where $S_{n}(k)$ stands for the

Received by the editors February 22, 1996.

1991 Mathematics Subject Classification. Primary 16S32; Secondary 16E30, 13N10.

Key words and phrases. Weyl algebra, $\mathcal{D}$-module, characteristic variety, Ext-groups.

(c)1997 American Mathematical Society 
homogeneous component of degree $k$ of $S_{n}$. If $d \in B_{k} \backslash B_{k-1}$, then we say that its degree is $\operatorname{deg}(d)=k$ and that its principal symbol is $\sigma(d)=\sigma_{k}(d)$.

Let $M$ be a finitely generated left $A_{n}$-module. A good filtration of $M$ is a filtration $\mathcal{F}$, associated to the Bernstein filtration, such that $g^{\mathcal{F}} M$ is finitely generated over $S_{n}$. Let $\mathrm{Ch}(M)$ denote the variety corresponding to the zeroes of the annihilator of $g r^{\mathcal{F}} M$ in $\mathbb{C}^{2 n}$. It is an invariant of $M$, called its characteristic variety. Note that $\mathrm{Ch}(M)$ is a homogeneous variety. The codimension of a module is the codimension of its characteristic variety.

Let $\omega$ be the standard symplectic form of $\mathbb{C}^{2 n}$. It can be used to define an isomorphism $\mathcal{I}: \mathbb{C}^{\star} \rightarrow \mathbb{C}$. If $f, g \in S_{n}$, then the Poisson bracket of $f$ and $g$ at $p \in \mathbb{C}^{2 n}$ is defined by

$$
\{f, g\}(p)=\omega(\mathcal{I} d f(p), \mathcal{I} d g(p)) .
$$

We will say that a variety $X$ of $\mathbb{C}^{2 n}$ is involutive if its ideal $\mathrm{I}(X)$ is closed under the Poisson bracket, in other words $\{\mathrm{I}(X), \mathrm{I}(X)\} \subseteq \mathrm{I}(X)$. It follows from the work of $\mathrm{O}$. Gabber in [6] that the characteristic variety of a finitely generated $A_{n}$-module is involutive. This severely restricts the varieties that can be characteristic varieties. For example, the dimension of an involutive variety of $\mathbb{C}^{2 n}$ cannot be less than $n$. An involutive variety of dimension $n$ in $\mathbb{C}^{2 n}$ is called Lagrangian.

Following [1], we shall say that a homogeneous involutive variety of $\mathbb{C}^{2 n}$ is minimal if it does not contain any proper homogeneous involutive subvariety. It follows from the comments above that an irreducible Lagrangian variety is necessarily minimal. In $\S 3$ we will see that there exist minimal varieties of smaller codimension.

Let $M$ be a finitely generated left $A_{n}$-module with a good filtration $\mathcal{F}$. If $W$ is an irreducible variety of $\mathbb{C}^{2 n}$, we will denote by $m_{W}(M)$ the multiplicity of $g r^{\mathcal{F}} M$ at $W$. The multiplicity of $M$ is the sum of the $m_{W}(M)$ with $W$ running over all minimal involutive homogeneous varieties of $\mathbb{C}^{2 n}$. The multiplicity of $M$ is finite if and only if the irreducible components of $\mathrm{Ch}(M)$ are minimal involutive homogeneous varieties. Note that the multiplicity is additive over short exact sequences.

A finitely generated left $A_{n}$-module is singular if it has finite multiplicity. The full subcategory of singular modules whose characteristic varieties are equidimensional of codimension $k$ will be denoted by $\mathcal{M}^{k}$. Thus $\mathcal{M}^{n}$ is the category of holonomic modules.

Actually, singular modules behave, in many ways, like holonomic modules. For example, the category of singular modules is closed under submodules, quotients and extensions; and its objects have finite length. The same is true of $\mathcal{M}^{k}$. Furthermore, if $M$ is an object of $\mathcal{M}^{k}$, then

1. $\operatorname{Ext}^{j}\left(M, A_{n}\right)=0$ if $j \neq k$;

2. $\operatorname{Ext}^{k}\left(M, A_{n}\right)$ is a right module with the same characteristic variety as $M$;

3. $\operatorname{Ext}^{k}\left(\operatorname{Ext}^{k}\left(M, A_{n}\right), A_{n}\right) \cong M$.

For a proof see $[1, \S 3]$ and $[7$, Corollary V.11]. These properties allow one to apply the following theorem from [2, Ch. 2, Proposition 4.12] to the calculation of Ext groups of singular modules.

2.1 Theorem. Let $M$ be a finitely generated left $A_{n}$-module. If $\operatorname{Ext}^{j}\left(M, A_{n}\right)=0$ whenever $j \neq k$, then for any finitely generated left $A_{n}$-module $N$

1. $\operatorname{Ext}^{j}(M, N)=0$ if $j>k$;

2. $\operatorname{Ext}^{j}(M, N) \cong \operatorname{Tor}_{k-j}\left(\operatorname{Ext}^{k}\left(M, A_{n}\right), N\right)$ for $0 \leq j \leq k$. 
If both $M$ and $N$ are singular, we can sharpen this result into a duality theorem for Ext groups. Let $\tau$ stand for the standard transposition of $A_{n}$, defined by $\tau\left(x^{\alpha} \partial^{\beta}\right)=(-1)^{|\beta|} \partial^{\beta} x^{\alpha}$. Note that $\tau$ determines an automorphism, to be denoted by $\tau^{\star}$, of $\mathbb{C}^{2 n}$, which maps $y_{i}$ onto itself and $y_{i+n}$ onto $-y_{i+n}$, for $1 \leq i \leq n$. Thus, if a variety is minimal involutive homogeneous of codimension $k$, then so is its image under $\tau^{\star}$. Now let $N$ be a finitely generated right $A_{n}$-module. Denote by $N^{\tau}$ the left $A_{n}$-module obtained from $N$ by transposing its action using $\tau$. Then $M^{\star}=\left(\operatorname{Ext}^{k}\left(M, A_{n}\right)\right)^{\tau}$ defines a functor of $\mathcal{M}^{k}$ into itself which satisfies $\left(M^{\star}\right)^{\star} \cong M$.

2.2 Corollary. Let $M$ and $N$ be objects of $\mathcal{M}^{t}$ and $\mathcal{M}^{s}$, respectively. Then

$$
\operatorname{Ext}^{j}(M, N) \cong \operatorname{Ext}^{s-t+j}\left(N^{\star}, M^{\star}\right)
$$

if $t-s \leq j \leq t$, and zero otherwise.

Proof. By Theorem 2.1, $\operatorname{Ext}^{j}(M, N)=0$ if $j>t$ and

$$
\operatorname{Ext}^{j}(M, N) \cong \operatorname{Tor}_{t-j}\left(\operatorname{Ext}^{t}\left(M, A_{n}\right), N\right)
$$

if $0 \leq j \leq t$. However, if $j<t-s$, then $t-j>s$. Since $N$ has projective dimension $s$, it follows that $\operatorname{Ext}^{j}(M, N)=0$, in this case. Thus we need only consider the case $t-s \leq j \leq t$. But

$$
\operatorname{Tor}_{t-j}\left(\operatorname{Ext}^{t}\left(M, A_{n}\right), N\right) \cong \operatorname{Tor}_{t-j}\left(N^{\tau}, M^{\star}\right) .
$$

Since $N^{\tau} \cong \operatorname{Ext}^{t}\left(N^{\star}, A_{n}\right)$, the result follows from another application of Theorem 2.1 .

This allows us to calculate Ext groups directly in a number of special cases.

2.3 Proposition. Let $M$ and $N$ be objects of $\mathcal{M}^{t}$ and $\mathcal{M}^{s}$, respectively. If $t \geq s$ and $j \leq t-s$, then $\operatorname{Ext}^{j}(M, N)$ is a finite dimensional complex vector space.

Proof. If $j<t-s$ then $\operatorname{Ext}^{j}(M, N)=0$ and there is nothing to prove. Assume that $j=t-s$. By Corollary 2.2

$$
\operatorname{Ext}^{t-s}(M, N) \cong \operatorname{Hom}_{A_{n}}\left(N^{\star}, M^{\star}\right) .
$$

Since singular modules have finite length, the result follows by Quillen's Lemma; see for example [13, Theorem 9.5.5].

It follows from [11] (see also [3]) that the characteristic variety of an irreducible $A_{n}$-module is equidimensional. Hence a singular irreducible module belongs to $\mathcal{M}^{k}$ for some $1 \leq k \leq n$. Let $M$ and $N$ be singular irreducible modules of codimensions $t$ and $s$, respectively. If $t>s+1$ then $\operatorname{Ext}^{1}(M, N)=0$. If $t=s+1$, then

$$
\operatorname{Ext}^{1}(M, N) \cong \operatorname{Hom}_{A_{n}}\left(N^{\star}, M^{\star}\right)=0 .
$$

Thus $\operatorname{Ext}^{1}(M, N)$ vanishes for $t>s$. This implies that there is no GK-critical $A_{n}$-module of length 2 that is singular. However, Perets has shown in [14] that GK-critical $A_{n}$-modules of length 2 do exist. The methods used here can be applied to give examples of such modules, as will be shown in a forthcoming paper.

2.4 Corollary. Let $M$ be a holonomic left $A_{n}$-module. If $N$ is any singular $A_{n^{-}}$ module, then $\operatorname{Ext}^{1}(M, N)$ is a finite dimensional complex vector space. 
Proof. Since singular modules have finite length, it is enough to check the result when $M$ and $N$ are irreducible. Suppose that $N$ has codimension $k$, for some $1 \leq k \leq n$. If $k<n$, the result follows from Proposition 2.3. If $k=n$, it follows from [2, Theorems 2.7.15 and 1.6.6].

The behaviour of Ext in the range $j>t-s$ is totally different, as shown by the next theorem. Recall that if $J$ is a left ideal of $A_{n}$, then its symbol ideal is $\sigma(J)=\sum_{k \geq 0} \sigma_{k}\left(J \cap B_{k}\right)$. This is an ideal of $A_{n}$, and $\operatorname{Ch}\left(A_{n} / J\right)=\mathcal{Z}(\sigma(J))$, the set of zeroes of $\sigma(J)$ in $\mathbb{C}^{2 n}$.

2.5 Theorem. Let $n \geq 2$ and $k \geq 1$ be integers. Suppose that $d \in A_{n}$ is an operator of degree $k$ and $J$ is a left ideal of $A_{n}$. If

1. the variety $\mathcal{Z}(\sigma(J)) \not \subset \mathcal{Z}\left(\sigma_{k}(d)\right)$, and

2. the symbol ideal of $J$ is prime, then $\operatorname{Ext}^{1}\left(A_{n} / A_{n} d, A_{n} / J\right)$ is an infinite dimensional vector space.

Proof. Since $A_{n} / A_{n} d$ has codimension 1, it follows from Theorem 2.1 that

$$
\operatorname{Ext}^{1}\left(A_{n} / A_{n} d, A_{n} / J\right) \cong A_{n} / d A_{n} \otimes_{A_{n}} A_{n} / J \cong A_{n} /\left(d A_{n}+J\right) .
$$

The embedding of $B_{r}$ into $A_{n}$ induces an injective map

$$
Q_{r}=B_{r} /\left(B_{r} \cap\left(d A_{n}+J\right)\right) \rightarrow A_{n} /\left(d A_{n}+J\right) .
$$

But $Q_{r} / Q_{r-1}$ is isomorphic, as a vector space, to the quotient of $B_{r} / B_{r-1}=S_{n}(r)$ by the subspace

$$
\frac{B_{r} \cap\left(d A_{n}+J\right)}{B_{r-1} \cap\left(d A_{n}+J\right)} .
$$

We claim that for $r>k$, this subspace is isomorphic to $S_{n}(r-k) \sigma_{k}(d)+\sigma_{r}\left(J \cap B_{r}\right)$. To prove this it is enough to show that the image of $B_{r} \cap\left(d A_{n}+J\right)$ under the symbol $\operatorname{map} \sigma_{r}$ is $S_{n}(r) \sigma_{r-k}(d)+\sigma_{r}\left(J \cap B_{r}\right)$.

Let us calculate $B_{r} \cap\left(d A_{n}+J\right)$ when $r>k$. Clearly

$$
d B_{r-k}+\left(J \cap B_{r}\right) \subseteq B_{r} \cap\left(d A_{n}+J\right) .
$$

Now let $b \in B_{r}$ and suppose that there exist $a \in A_{n}$ and $q \in J$ such that $b=d a+q$. By [4, Lemma 2.3] we can assume that $\sigma(a) \notin \sigma(J)$. If $r<s=$ $\max \{\operatorname{deg}(\operatorname{da}), \operatorname{deg}(q)\}$, then

$$
\sigma_{k}(d) \sigma_{s-k}(a)+\sigma_{s}(q)=0 .
$$

If $\operatorname{deg}(d a)<s=\operatorname{deg}(q)$, then $\sigma_{s}(q)=0$. Thus $q=0$ and $b \in d B_{r-k}$. Therefore, we can assume that $s=\operatorname{deg}(d a)$. Since $\sigma(J)$ is prime and $\sigma_{s-k}(a)=\sigma(a) \notin \sigma(J)$, it follows that $\sigma_{k}(d) \in \sigma(J)$, which contradicts (1). Hence $r=\max \{\operatorname{deg}(d a), \operatorname{deg}(q)\}$, and so $b \in d B_{r-k}+\left(J \cap B_{r}\right)$. Therefore $d B_{r-k}+\left(J \cap B_{r}\right)=B_{r} \cap\left(d A_{n}+J\right)$. The claim follows immediately from this identity.

In particular, if $r>k$, then

$$
Q_{r} / Q_{r-1} \cong S_{n}(r) /\left(S_{n}(r-k) \sigma_{k}(d)+\sigma_{r}\left(J \cap B_{r}\right)\right) .
$$

Denote the latter by $G(r)$. The $S_{n}$-module

$$
G=S_{n} /\left(S_{n} \sigma_{k}(d)+\sigma(J)\right)
$$


is graded by the $G(r)$, for $r \geq 0$. An argument similar to [9, Theorem I.7.5] shows that there exists a polynomial $\chi(t)$ with integral coefficients such that for $r \gg 0$

$$
\sum_{i=0}^{r} \operatorname{dim}_{\mathbb{C}} G(i)=\chi(r)
$$

The degree of $\chi(t)$ is equal to the dimension of the variety $\mathcal{Z}\left(S_{n} \sigma_{k}(d)+\sigma(J)\right)$ in $\mathbb{C}^{2 n}$.

However, $\mathcal{Z}\left(\sigma_{k}(d)\right)$ and $\mathcal{Z}(\sigma(J))$ are both homogeneous varieties of $\mathbb{C}^{2 n}$, hence their intersection is non-empty. Since $\mathcal{Z}(\sigma(J))$ is involutive, its dimension is at least $n$. Thus, by [9, Proposition I.7.1], the intersection of these two varieties has dimension at least $(2 n-1)+n-2 n=n-1$. But this is the degree of $\chi(t)$. Since $n \geq 2$, we conclude that $\chi(t)$ has degree at least 1 .

Collecting the previous results, we have that for $r \gg 0$

$$
\operatorname{dim}_{\mathbb{C}} \operatorname{Ext}^{1}\left(A_{n} / A_{n} d, A_{n} / J\right) \geq \chi(r)-c
$$

where $c$ is a constant that corresponds to the sum of the dimensions of the first $k+1$ homogeneous components of $G$. Since $\chi(t)$ is a polynomial of degree at least 1 , it follows that $\operatorname{Ext}^{1}\left(A_{n} / A_{n} d, A_{n} / J\right)$ is infinite dimensional.

To apply Theorem 2.5 we need examples of left ideals $J$ of $A_{n}$ whose symbol ideal is prime. These are easy to construct. For example, suppose that $I$ and $J$ are left ideals of $A_{m}$ and $A_{n}$, respectively, whose symbol ideals are prime. Then the same holds for the $A_{m+n}$-module $M \otimes_{K} N$. In $\S 3$, we will construct singular modules using external products. For the next corollary we retain the notation and hypotheses of Theorem 2.5. The corollary follows from Theorem 2.5 and Corollary 2.2 .

2.6 Corollary. Let $n \geq 2$. Suppose that $M=A_{n} / A_{n} d$ and $N=A_{n} / J$ are singular left $A_{n}$-modules of codimensions 1 and $k>1$, respectively. Then $\operatorname{Ext}^{1}(M, N)$ and $\operatorname{Ext}^{k}(N, M)$ are infinite dimensional vector spaces.

Collecting previous results, we can determine when the $\operatorname{Ext}^{1}$ groups of singular modules are finite dimensional for $n=2$. The following notation makes the result easier to state. Let $\mathbb{V}$ be the category of complex vector spaces and let

$$
\mathcal{F}: \mathcal{M}^{t} \times \mathcal{M}^{s} \rightarrow \mathbb{V}
$$

be a bifunctor. We say that $\mathcal{F}$ is finite dimensional if $\mathcal{F}(M, N)$ is a finite dimensional vector space for all objects $M$ of $\mathcal{M}^{t}$ and $N$ of $\mathcal{M}^{s}$.

2.7 Corollary. Let $n=2$ and $1 \leq t, s \leq 2$. The bifunctor

$$
\operatorname{Ext}^{1}(\cdot, \cdot): \mathcal{M}^{t} \times \mathcal{M}^{s} \rightarrow \mathbb{V}
$$

is finite dimensional if and only if $t=2$.

To put corollary 2.7 in perspective, recall that Hom is finite dimensional for all $1 \leq t, s \leq 2$, by [13, Theorem 9.5.5]. On the other hand, there is no analogue of Corollary 2.7 for $\mathrm{Ext}^{2}$, since, choosing $d \in A_{2}$ such that $A_{2} / A_{2} d$ has a minimal involutive homogeneous characteristic variety, we have that

$$
\operatorname{Ext}^{2}\left(\mathbb{C}\left[x_{1}, x_{2}\right], A_{2} / A_{2} d\right) \cong \operatorname{Ext}^{1}\left(A_{2} / A_{2} d, \mathbb{C}\left[x_{1}, x_{2}\right]\right)
$$

has infinite dimension by Theorem 2.5. Note also that if $M$ and $N$ are singular of codimension 1 and their characteristic varieties are not equal then $\operatorname{Ext}^{1}(M, N)$ 
may be infinite dimensional. The case of equal characteristic varieties is discussed in $[4$, Theorem 4.5].

\section{Codimension 2}

To understand the behaviour of extensions of singular modules of codimension 2 , it will be necessary to construct a family of modules with this codimension. But first we must find examples of minimal homogeneous involutive varieties of codimension 2. This makes use of some results of Bernstein and Lunts, which we now describe.

Bernstein and Lunts proved in [1] that when $n=2$, 'most' homogeneous hypersurfaces of $\mathbb{C}^{2 n}$ are minimal. This was later generalized to $n \geq 2$ by Lunts in [12]. Since we want to apply their result, it is necessary to state it in greater detail. For $f \in S_{n}(k)$, define $h_{f}$ to be the corresponding Hamiltonian vector field of $\mathbb{C}^{2 n}$. For $g \in S_{n}$, we have that $h_{f}(g)=\{f, g\}$. A property $\mathbf{P}$ holds generically in $S_{n}(k)$ if the set

$$
\left\{g \in S_{n}(k): g \text { does not satisfy } \mathbf{P}\right\}
$$

is contained in a countable union of hypersurfaces of $S_{n}(k)$. Following the usual shorthand, we will say that if $f$ is generic in $S_{n}(k)$, then $\mathbf{P}$ holds. The next result is a consequence of $[12$, Theorem 1$]$.

3.1 Theorem. Let $n \geq 2$ and $k \geq 4$. If $f$ is a generic element of $S_{n}(k)$, then a proper subvariety of the hypersurface $\mathcal{Z}(f)$ stable under $h_{f}$ must have dimension at most 1 .

In particular, if $f$ is generic in $S_{n}(k)$, the hypersurface $\mathcal{Z}(f)$ is a minimal homogeneous involutive variety. In the above form the result also allows us to construct minimal homogeneous involutive varieties of codimension 2. Following the usual notation, we will say that, for $1 \leq i \leq n$, the variables $y_{i}$ and $y_{i+n}$ are conjugate, since $\left\{y_{i}, y_{i+n}\right\}=1$.

3.2 Theorem. Suppose that $n \geq 3$ and that $k \geq 4$. Let $f$ be a generic homogeneous polynomial in the first $n-1$ pairs of conjugate variables. The subvariety $W$ of $\mathbb{C}^{2 n}$ with equations $f=y_{2 n}=0$ is a minimal homogeneous involutive variety of dimension $2 n-2$.

Proof. It is clear that $W$ is involutive, since the ideal generated by $f$ and $y_{2 n}$ is prime in $S_{n}$ and $\left\{f, y_{2 n}\right\}=0$. We must show that $W$ is minimal. The proof is by contradiction. Suppose that $Y$ is an irreducible homogeneous involutive subvariety of $\mathbb{C}^{2 n}$ properly contained in $W$. In particular, $Y$ has codimension at least 3 . Denote by $\mathbb{C}^{2 n-2}$ the linear subspace with equations $y_{n}=y_{2 n}=0$. Since $f$ is a polynomial in the first $n-1$ pairs of conjugate variables, the Hamiltonian vector field $h_{f}$ of $\mathbb{C}^{2 n}$ restricts to the corresponding Hamiltonian field in $\mathbb{C}^{2 n-2}$. For the same reason, the hyperplane $H$ with equation $y_{n}=0$ is stable under $h_{f}$. But $Y$ is an involutive subvariety whose ideal contains $f$; hence $Y$ is also stable under $h_{f}$. Thus $Y \cap H$ is stable under $h_{f}$.

But $Y \cap H$ can be viewed as a subvariety of $\mathbb{C}^{2 n-2}$, contained in $\mathcal{Z}(f)$. Since it is stable under $h_{f}$, it follows from Theorem 3.1 that either $\operatorname{dim}(Y \cap H) \leq 1$ or $Y \cap H=\mathcal{Z}(f) \cap \mathbb{C}^{2 n-2}$ as subvarieties of $\mathbb{C}^{2 n-2}$. In the first case, using [9, Proposition I.7.1] and the fact that $Y$ and $H$ are homogeneous subvarieties of $\mathbb{C}^{2 n}$, 
we have that

$$
\operatorname{dim}(Y \cap H) \geq \operatorname{dim}(Y)+\operatorname{dim}(H)-2 n=\operatorname{dim}(Y)-1 .
$$

Since $Y$ is involutive, its dimension is at least $n$. Therefore

$$
n-1 \leq \operatorname{dim}(Y \cap H) \leq 1,
$$

and so $n \leq 2$, a contradiction. Thus we must have that $Y \cap H=\mathcal{Z}\left(f, y_{n}, y_{2 n}\right)$ in $\mathbb{C}^{2 n}$. But $\mathcal{Z}\left(f, y_{n}, y_{2 n}\right)$ has codimension 3 in $\mathbb{C}^{2 n}$. Since $Y$ is irreducible, we conclude that $Y=\mathcal{Z}\left(f, y_{n}, y_{2 n}\right)$. However an ideal that contains $y_{n}$ and $y_{2 n}$ cannot be involutive, since $\left\{y_{n}, y_{2 n}\right\}=1$. This contradicts the fact that $Y$ is involutive, and completes the proof.

It is easy to construct $A_{n}$-modules whose characteristic variety is $W$. Let $M$ be an $A_{n-1}$-module with characteristic variety $\mathcal{Z}(f)$. The external product $M \otimes_{\mathbb{C}} \mathbb{C}\left[x_{n}\right]$ has $W$ for its characteristic variety. Hence $M \otimes_{\mathbb{C}} \mathbb{C}\left[x_{n}\right]$ is singular of codimension 2.

3.3 Theorem. Let $n \geq 3$ and $k \geq 4$ be integers. Suppose that $d \in A_{n-1}$ is an operator of degree $k$ whose principal symbol is generic in $S_{n-1}(k)$. Let $J$ be a left ideal of $A_{n-1}$. If $\operatorname{Ext}^{1}\left(A_{n-1} / A_{n-1} d, A_{n-1} / J\right)$ is an infinite dimensional vector space, then so is $\operatorname{Ext}^{1}\left(A_{n} /\left(A_{n} d+A_{n} \partial_{n}\right), A_{n} /\left(A_{n} J+A_{n} \partial_{n}\right)\right)$.

Proof. We have that

$$
M=A_{n} /\left(A_{n} d+A_{n} \partial_{n}\right) \cong A_{n-1} / A_{n-1} d \otimes_{\mathbb{C}} \mathbb{C}\left[x_{n}\right] .
$$

By Theorem 3.2, $\mathrm{Ch}(M)=W$ is a minimal involutive homogeneous variety. Thus $M$ is singular of codimension 2. In particular, $\operatorname{Ext}^{j}\left(M, A_{n}\right)=0$ whenever $j \neq 2$. It is easy to check that $\operatorname{Ext}^{2}\left(M, A_{n}\right) \cong A_{n} /\left(d A_{n}+\partial_{n} A_{n}\right)$ has a free resolution of the form

$$
0 \rightarrow A_{n} \stackrel{\alpha}{\longrightarrow} A_{n}^{2} \stackrel{\beta}{\rightarrow} A_{n} \rightarrow \operatorname{Ext}^{2}\left(M, A_{n}\right) \rightarrow 0 .
$$

Writing the elements of $A_{n}^{2}$ as column vectors, we have that $\alpha$ and $\beta$ are represented by the matrices

$$
\alpha=\left(\begin{array}{c}
d \\
\partial_{n}
\end{array}\right) \text { and } \beta=\left(\partial_{n},-d\right) \text {. }
$$

Now let $N=A_{n} /\left(A_{n} J+A_{n} \partial_{n}\right) . \operatorname{Thus}_{\operatorname{Ext}^{1}}(M, N) \cong \operatorname{Tor}_{1}\left(\operatorname{Ext}^{2}\left(M, A_{n}\right), N\right)$, by Theorem 2.1. Tensoring (3.4) with $N$, we get the complex

$$
0 \rightarrow N \stackrel{\alpha}{\rightarrow} N^{2} \stackrel{\beta}{\rightarrow} N \rightarrow 0
$$

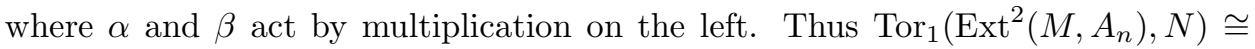
$\operatorname{ker}(\beta) / \operatorname{im}(\alpha)$. Denote by $\bar{a}$ the image of $a \in A_{n}$ in $N$. Then

$$
\alpha(\bar{a})=\left(\begin{array}{c}
d \bar{a} \\
\partial_{n} \bar{a}
\end{array}\right) .
$$

Choose $b \in A_{n-1}$. Clearly

$$
(\bar{b}) \in \operatorname{ker} \beta
$$


This element will be in $\operatorname{im}(\alpha)$ if and only if $b \in d A_{n}+A_{n} J+A_{n} \partial_{n}$. Since we are assuming that $b \in A_{n-1}$, and both $d$ and $J$ are contained in $A_{n-1}$, it follows that $b \in d A_{n-1}+J$. Thus we have an injective linear map of vector spaces

$$
\operatorname{Ext}^{1}\left(A_{n-1} / A_{n-1} d, A_{n-1} / J\right) \rightarrow \operatorname{Tor}_{1}\left(\operatorname{Ext}^{2}\left(M, A_{n}\right), N\right)
$$

defined by mapping $b$ onto $(\bar{b}, \overline{0})$. If $\operatorname{Ext}^{1}\left(A_{n-1} / A_{n-1} d, A_{n-1} / J\right)$ is a vector space of infinite dimension, so is $\operatorname{Tor}_{1}\left(\operatorname{Ext}^{2}\left(M, A_{n}\right), N\right)$. Hence $\operatorname{Ext}^{1}(M, N)$ is infinite dimensional in this case.

We may now state a result similar to Corollary 2.6 when $n=3$. It is obtained by combining Theorems 2.5 and 3.3 and Corollary 2.2.

3.5 Corollary. Let $n=3$ and $1 \leq t, s \leq 3$. The bifunctor

$$
\operatorname{Ext}^{1}(\cdot, \cdot): \mathcal{M}^{t} \times \mathcal{M}^{s} \rightarrow \mathbb{V}
$$

is finite dimensional if and only if $t=3$.

\section{Final COMments}

The real challenge is to solve the following problem.

4.1 Problem. Let $M$ be a singular $A_{n}$-module and let $1 \leq k \leq n$. Is it true that if the functor

$$
\operatorname{Ext}^{1}(M, \cdot): \mathcal{M}^{k} \rightarrow \mathbb{V}
$$

is finite dimensional then $M$ is holonomic?

To find a positive solution to problem 4.1 using the approach of this paper one has to sort out the following points:

1. Remove the ' $\sigma(J)$ is prime' condition from Theorem 2.5.

2. Construct minimal involutive homogeneous varieties of codimension $>2$ in $\mathbb{C}^{2 n}$.

The second point leads into further problems. For let us not forget that our aim in constructing these varieties is to produce singular modules of codimension $>$ 2. However there is no known criterion to determine whether a given involutive homogeneous variety is a characteristic variety or not. One way to remove this obstacle is to show that the following problem has an affirmative answer.

4.2 Problem. Let $X$ and $Y$ be minimal involutive homogeneous varieties of $\mathbb{C}^{2 m}$ and $\mathbb{C}^{2 n}$, respectively. Is $X \times Y$ a minimal involutive homogeneous variety of $\mathbb{C}^{2(n+m)}$ ?

Assuming that $X$ and $Y$ are characteristic varieties, there exist $M$ and $N$, finitely generated modules over $A_{m}$ and $A_{n}$ respectively, such that $\operatorname{Ch}(M)=X$ and $\operatorname{Ch}(N)=Y$. Thus $M \otimes_{\mathbb{C}} N$ is a finitely generated $A_{m+n}$-module whose characteristic variety is $X \times Y$. If the latter is minimal, we are done. It is not difficult to use the method of the proof of Theorem 3.2 to show the following related result.

4.3 Proposition. Let $n>m+1 \geq 2$ and $k \geq 4$ be integers. Suppose that $f$ is generic in $S_{n}(k)$ and that $X$ is an involutive homogeneous subvariety of $\mathbb{C}^{2 m}$. Then $\mathcal{Z}(f) \times X$ contains a minimal homogeneous involutive subvariety of codimension $d$, where $1<d<n+m$. 
Finally, Theorem 2.1 is useful for calculating extensions even when the modules are not singular. Here is an example. Let $f \in \mathbb{C}\left[x_{1}, \ldots, x_{n}\right]$ and let $s$ be an indeterminate that commutes with the operators of $A_{n}$. Put $A_{n}[s]=A_{n} \otimes_{\mathbb{C}} \mathbb{C}[s]$ and write

$$
M_{f}=A_{n}[s] f^{s} .
$$

The following properties of $M_{f}$ are proved in [10] (see also [8]).

4.4 Proposition. The module $M_{f}$ is finitely generated over $A_{n}$ and satisfies:

1. $\operatorname{Ext}^{j}\left(M_{f}, \mathcal{D}(X)\right)=0$ unless $j=n-1$.

2. The characteristic variety $W_{f}$ of $M_{f}$ with respect to the order filtration is the closure, in the Zariski topology, of the set

$$
\left\{(x, s d \log f(x)) \in \mathbb{C}^{n} \times \mathbb{C}^{n}: f(x) \neq 0, s \in \mathbb{C}\right\} .
$$

Note that $M_{f}$ is an $A_{n}$-module of Krull dimension 1, by [5, Theorem 2.6]. Hence it cannot be singular. However this module has a well defined dual, by Proposition 4.4(1).

4.5 Proposition. Suppose that $n \geq 2$. Let $Y$ be an irreducible smooth subvariety of $\mathbb{C}^{n}$ and let $f \in \mathbb{C}\left[x_{1}, \ldots, x_{n}\right]$. Assume that

1. the codimension of $Y$ is at least 2 , and

2. $Y$ is not contained in the hypersurface $f=0$ of $X$.

If $N$ is a finitely generated $A_{n}$-module whose characteristic variety (with respect to the order filtration) is the conormal bundle $\mathrm{T}_{Y}^{\star} \mathbb{C}^{n}$, then $\operatorname{Ext}^{1}\left(N, M_{f}\right)=0$.

Proof. Note that although $M_{f}$ is not singular, we can still apply Theorem 2.2. Besides, transposition does not affect the characteristic variety with respect to the order filtration. Hence

$$
\operatorname{Ext}^{1}\left(N, M_{f}\right) \cong \operatorname{Hom}_{A_{n}}\left(M_{f}^{\star}, N^{\star}\right) .
$$

Let $p \in Y$ and suppose that $f(p) \neq 0$. Denote by $\pi$ the projection of $T^{\star} \mathbb{C}^{n}$ on its base. Then $\pi^{-1}(p) \cap \mathrm{T}_{Y}^{\star} X$ has dimension at least 2. On the other hand, $\pi^{-1}(p) \cap W_{f}$ has dimension 1. Hence $\mathrm{T}_{Y}^{\star} \mathbb{C}^{n} \not \subset W_{f}$. Thus $\operatorname{Hom}_{A_{n}}\left(M_{f}^{\star}, N^{\star}\right)=0$, which is what we wanted to prove.

This result suggests that some of the computations in the paper may be extended to categories of modules that do not have finite length.

\section{REFERENCES}

1. I.N. Bernstein and V. Lunts, On non-holonomic irreducible D-modules, Invent. Math. 94 (1988), 223-243. MR 90b:58247

2. J.-E. Björk, Rings of differential operators, North-Holland Publishing Company, Amsterdam, 1979. MR 82g:32013

3. J.-E. Björk, Filtered noetherian rings, Noetherian Rings and Their Applications (L.W. Small, ed.), Mathematical Surveys and Monographs 24, American Mathematical Society, Providence, 1987. MR 89c: 16018

4. S.C. Coutinho, Modules of codimension one over Weyl algebras, J. Algebra 177 (1995), 102114. MR 96i: 16041

5. S.C. Coutinho, Krull dimension of modules and involutive ideals, Proc. Amer. Math. Soc. 123 (1995), 1647-1654. MR 95g:16023

6. O. Gabber, The integrability of the characteristic variety, Amer. J. Math. 103 (1981), 445468. MR 82j:58104 
7. V. Ginsburg, Characteristic varieties and vanishing cycles, Invent. Math. 84 (1986), 327-402. MR 87j:32030

8. A. Gyoja, Theory of pre-homogeneous vector spaces without regularity condition, Publ. RIMS, Kyoto Univ. 27 (1991), 861-922. MR 93f:22018

9. R. Hartshorne, Algebraic geometry, Graduate Texts in Mathematics 52, Springer-Verlag, New York-Heidelberg-Berlin, 1977. MR 57:3116

10. M. Kashiwara, B-functions and holonomic systems: rationality of roots of B-functions, Invent. Math. 47 (1976), 33-53. MR 55:3309

11. T. Levasseur, Equidimensionalité de la variété caractéristique, exposé de O. Gabber rédigé par T. Levasseur, unpublished.

12. V. Lunts, Algebraic varieties preserved by generic flows, Duke Math. J. 58 (1989), 531-554. MR 91a:32015

13. J. C. McConnell and J. C. Robson, Noncommutative noetherian rings, John Wiley and Sons, New York, 1987. MR 89j:16023

14. G. Perets, d-critical modules of length 2 over Weyl algebras, Israel J. Math. 83 (1993), 361368. MR 94m:17007

Instituto de Matemática, Universidade Federal do Rio de Janeiro, P.O. Box 68530, 21945-970, Rio DE JANeiro, RJ, BraziL

E-mail address: collier@impa.br 